\title{
Human papillomavirus vaccine: waiting for a miracle
}

C $M A J$ is publishing this special issue on the human papillomavirus (HPV) vaccine in part to acknowledge those researchers who developed the HPV vaccine and to acknowledge the pharmaceutical industry, which embarked on the long and difficult task of bringing these vaccines to market. Vaccines have never been considered lucrative from an industry perspective. However, from a public health perspective, they have prevented or eradicated many diseases. Indeed, the introduction of the polio vaccine to Canada in 1955 ended an annual horror of outbreaks. Now, the HPV vaccines, in combination with cervical cancer screening programs, have the potential to make cervical cancer deaths a bad memory. In this issue, we summarize the existing evidence, determine the number needed to be vaccinated and discuss policy-related concerns (Box I).

There is still a lot to do. Despite a great beginning, there are many unanswered questions pertaining to long-term efficacy, optimal dosing, overall effectiveness against HPV in the real world and optimal delivery modalities in high-risk and impoverished populations. ${ }^{1}$

While there is guarded optimism about the potential longterm benefit of the 2 new HPV vaccines, there is growing concern that vaccine development efforts may be mothballed in favour of more profitable pharmaceutical products. The high cost of vaccine development and limited markets, coupled with some people's vaccine angst, have conspired to make vaccine development unattractive, especially in comparison to "lifestyle" drugs, such as sildenafil - a surefire money-maker because of its widespread, regular use. Given the magnitude of investment, only a handful of large companies remain committed to vaccine research and development. Yet, as exemplified by the polio saga, vaccines can be remarkably efficacious in preventing diseases and demonstrably diminishing human suffering.

HPV vaccines hold a similar promise. However, they will need a helping hand. Cervical cancer has a predilection for the impoverished and disenfranchised in developed and developing countries. In the Western world, vaccination and cervical cancer screening programs for high-risk individuals, such as Aboriginal youth, street youth and people living in poverty-stricken areas, present significant barriers to eradicating cervical cancer. Worldwide, over $80 \%$ of cervical cancer deaths occur in developing countries, where 470000 cases are confirmed annually (approximating half the number of new cases of breast cancer). ${ }^{2}$

Developing countries must not be left behind. The delays and barriers that occurred in the fight to eradicate polio in Africa and Southeast Asia must not be replicated in the attack on cervical cancer with the HPV vaccine and cervical cancer screening programs. This fight will require more than money and vaccines; it will require country-specific implementation

\section{Box 1: In this issue of CMAJ}

- Brisson and colleagues estimate that the number needed to vaccinate against human papillomavirus (HPV) infection would be 8 to prevent an episode of genital warts and 324 to prevent a case of cervical cancer (page 464).

- A systematic review by Rambout and colleagues shows that prophylactic HPV vaccination is highly efficacious in preventing vaccine type-specific HPV infection and cervical disease. However, the authors identify a number of studydesign limitations and knowledge gaps (page 469).

- Ogilvie and colleagues demonstrate the feasibility of selfcollection of specimens for HPV testing among women who may not make full use of cytology screening programs and are thus at increased risk of cervical cancer (page 480).

- Lippman and colleagues argue against widespread implementation of vaccine programs. Rather, they suggest that there is no epidemic or urgency to implement a mass vaccination program (page 484).

- Dawar and colleagues review the epidemiology and disease outcomes of HPV infection and describe the clinical trials of 2 HPV vaccines (page 456).

- A patient information sheet about HPV is provided (page 462).

strategies, leadership, infrastructure and cultural sensitivity. Major pharmaceutical companies have much to contribute, because they have an abundance of leadership, management and logistics expertise, and administrative staff. CMAJ implores the vaccine industry to donate generously of its time and expertise to create a miracle. We encourage companies to work with key players, including local authorities, nongovernmental agencies and funders, so that HPV vaccines and cervical cancer screening programs can be rolled out in developing countries. The potential for a miracle is present: the tools (vaccine and screening) are here. Cervical cancer can be beaten.

\section{Noni MacDonald MD MSc}

Section Editor, Public Health, CMAJ

Paul C. Hébert MD MHSc

Editor-in-Chief, CMAJ

For the Editorial-Writing Team (Matthew Stanbrook, Barbara Sibbald, Ken Flegel and Amir Attaran)

\section{REFERENCES}

I. Public Health Agency of Canada. Canadian human papillomavirus vaccine research priorities workshop: final report. Can Commun Dis Rep 2006;32(Suppl I). Available: www.phac-aspc.gc.ca/publicat/ccdr-rmtc/o6vol32/32si/index.html (accessed 2007 July 26)

2. Stewart BW, Kleihues P. World cancer report. Geneva: WHO; 2003. Available: www.iarc.fr/WCR/ (accessed 2007 July 26). 